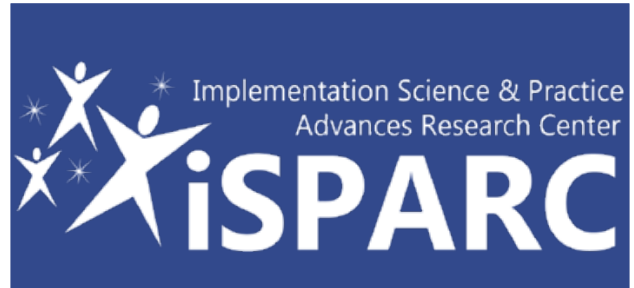

Tip Sheet

\title{
WHAT DO YOU KNOW ABOUT SERVING SOUTHEAST ASIAN IMMIGRANTS \& REFUGEES? Here are 5 Tips!
}

mmigrants and refugees from

Southeast Asia, including Laos, Cambodia, and Vietnam, do not easily talk about mental health conditions. Oftentimes symptoms of stress are not seen as psychological, but are attributed to other causes or described as physical ailments. Many Southeast Asian refugees have experienced trauma, sometimes severe, in their home countries prior to leaving or during their travel to the United States. Increasing your sensitivity to Southeast Asian traditions and culture can help you to better serve your clients.

Lastly, partnering with Cultural Brokers, culturally competent social workers, patient advocates, and organizations like the Southeast Asian Coalition of Central Massachusetts, Inc., can assist you in building a trusting relationship with your Southeast Asian clients.
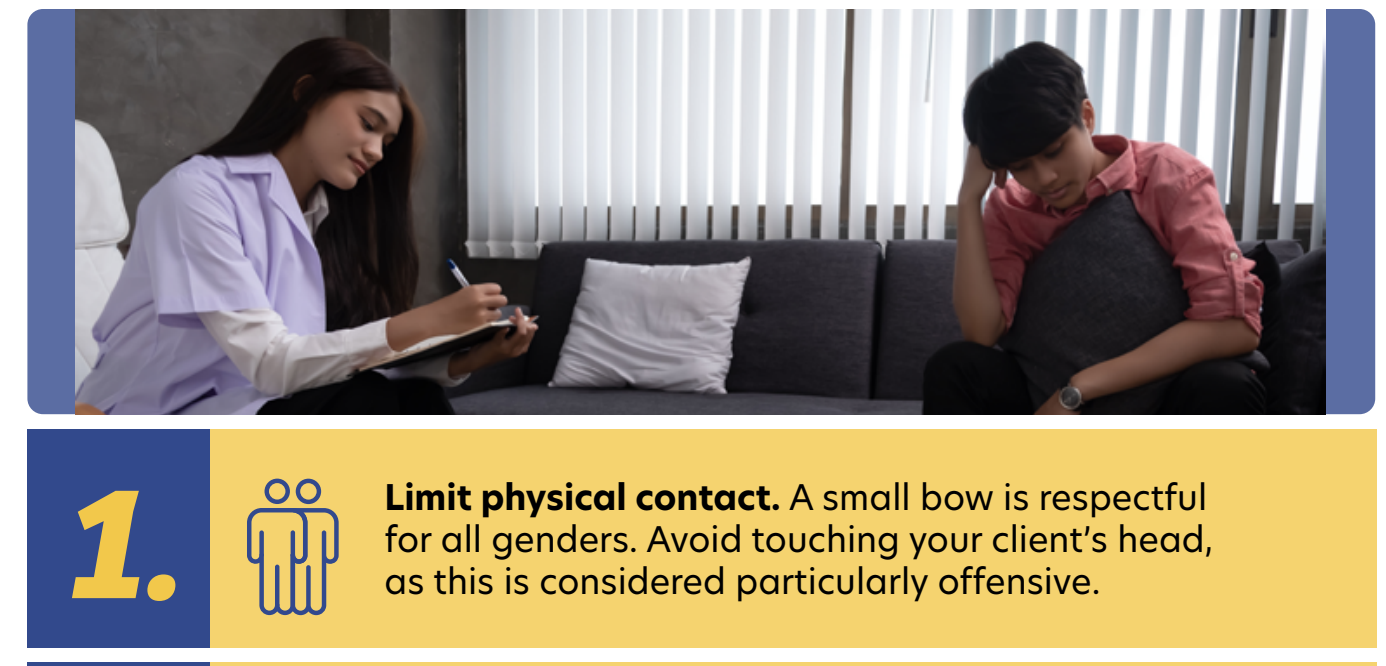

Limit physical contact. A small bow is respectful for all genders. Avoid touching your client's head, as this is considered particularly offensive.
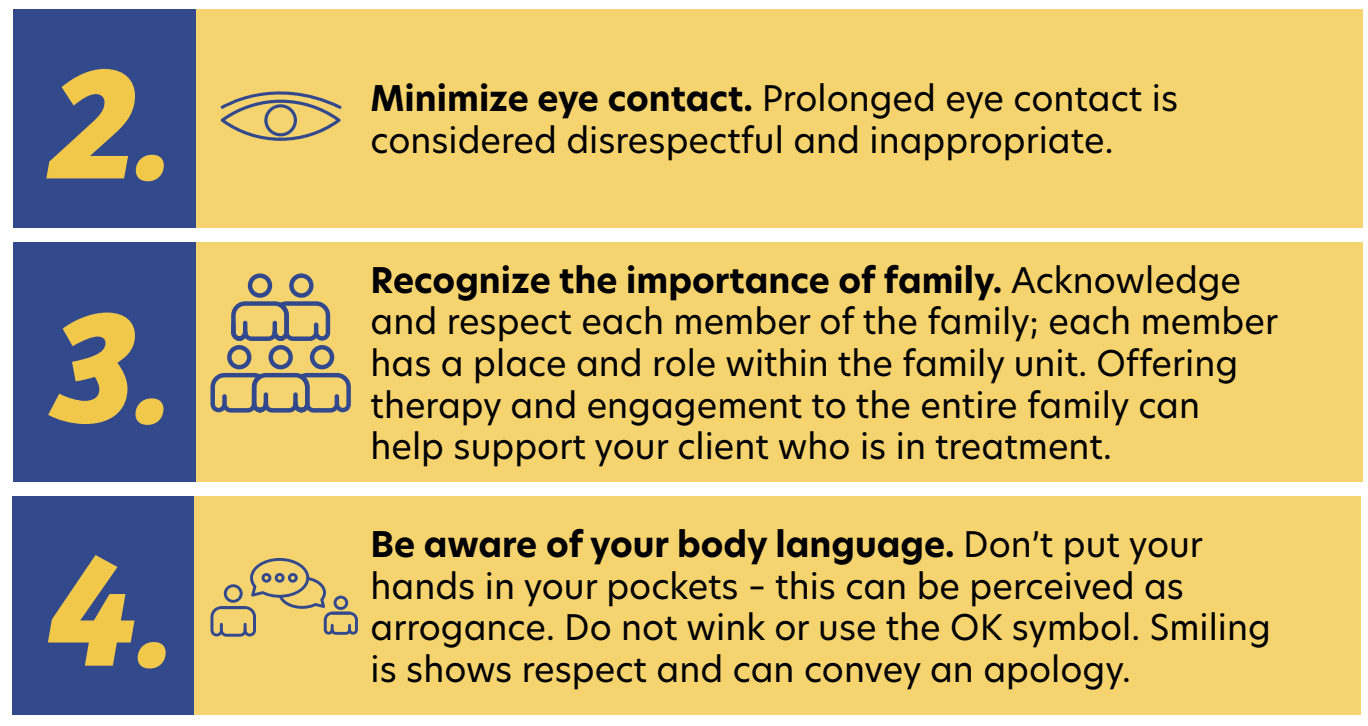

Minimize eye contact. Prolonged eye contact is considered disrespectful and inappropriate.
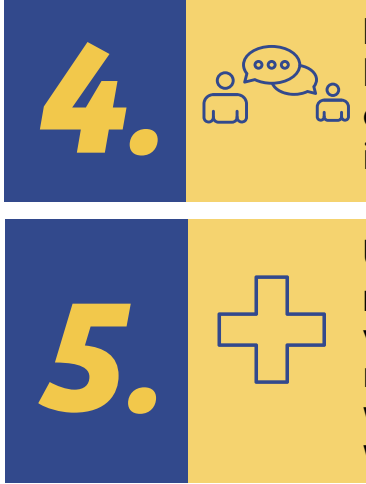

Be aware of your body language. Don't put your hands in your pockets - this can be perceived as arrogance. Do not wink or use the OK symbol. Smiling is shows respect and can convey an apology.

For more information on this topic, see iSPARC's Tips for Mental Health Providers Working with Southeast Asian Immigrants/Refugees.

\section{Like what you see? Visit iSPARC's website for more great content.}

